\title{
ANÁLISE ESPACIAL DA DISTRIBUIÇÃO DE CIGARRAS (Quesada gigas Oliver) EM POVOAMENTOS DE PARICÁ (Schizolobium amazonicum Huber ex Ducke) NA REGIÃO DE DOM ELISEU, PA ${ }^{1}$
}

\author{
Vicente Paulo Soares ${ }^{2}$, Luciano Zumerle Zaneti ${ }^{3}$, Nerilson Terra Santos ${ }^{4}$ e Helio Garcia Leite ${ }^{2}$
}

\begin{abstract}
RESUMO - O objetivo deste estudo foi avaliar, através da análise espacial de dados geográficos, a precisão de procedimentos de mapeamento das populações de cigarras (Quesada gigas Oliver) em povoamentos de paricá (Schizolobium amazonicum Huber ex Ducke), localizados no Município de Dom Eliseu, PA, mediante a aplicação de dois interpoladores (krigagem e inverso do quadrado da distância). Para tal, foram registradas amostras na intensidade de um ponto a cada $30 \mathrm{ha}$, onde se realizou a contagem do número de cigarras por planta (cigarras/árvore), obedecendo à estratégia de amostragem convencionalmente usada pela empresa proprietária do reflorestamento em estudo. As análises indicaram que a intensidade amostral empregada neste trabalho não foi suficiente para permitir a detecção de forte dependência espacial do número de cigarras/árvore nas áreas estudadas. Devido a essa baixa dependência espacial, os resultados dos dois métodos de interpolação (krigagem ordinária e inverso do quadrado da distância) apresentaram-se semelhantes. Já os deste trabalho indicaram que as estratégias de amostragem utilizadas devem ser melhoradas para gerar mapas de predição mais precisos da quantidade de cigarras/árvore nos reflorestamentos de paricá.
\end{abstract}

Palavras-chave: Paricá, cigarra e distribuição espacial.

\section{ANALYSIS OF THE SPATIAL DISTRIBUTION OF BUZZERS (Quesada gigas Oliver) IN PARICA STANDS (Schizolobium amazonicum Huber ex Ducke) IN DOM ELISEU-PA}

\begin{abstract}
The objective of this study was to evaluate the occurrence of buzzers (Quesada gigas Oliver) in Parica (Schizlobium amazonicum Huber ex Ducke) stands using spatial geographic data analysis, in Dom Eliseu, Para, Brazil, based on the application of two interpolators (kriging and distance square inverse).Thus, points were set up in a density of one point for every 30 hectares, with one tree sampled per point, obeying the sample strategy conventionally used by the company which owns the reforestation stand under study. The analysis indicated that the sampling intensity utilized in this work was not sufficient to allow a strong spatial dependence of the number of buzzers/tree in the study area. Due to this low spatial dependence, the results of both interpolation methods (ordinary kriging and distance square inverse) were similar. The results of this work indicate that the sampling strategies must be improved to generate better and more precise buzzer/tree prediction maps for the Parica reforestation stand.
\end{abstract}

Keywords: Paricá stands, buzzer, and spatial distribution.

\footnotetext{
${ }^{1}$ Recebido em 31.10.2006 e aceito para publicação em 20.02.2008.

${ }^{2}$ Departamento de Engenharia Florestal da Universidade Federal de Viçosa (UFV). E-mail : <vicente@ufv.br>.

${ }^{3}$ Programa de Pós-Graduação em Ciência Florestal da UFV. E-mail :<lucianozaneti@ yahoo.com.br>.

${ }^{4}$ Departamento de Informática da UFV. E-mail : <nsantos@ dpi.ufv.br>.
} 


\section{INTRODUÇÃO}

A cigarra (Quesada gigas Oliver) vem-se apresentando como uma praga em potencial para o paricá. Segundo Zanuncio (2004), em meados de 2002 cigarras da espécie Quesada gigas (Oliver) foram registradas em plantações de Schizolobium amazonicum (Huber ex Ducke) nos Municípios de Itinga do Maranhão, MA, e Paragominas, PA, atacando o sistema radicular das plantas e provocando danos severos em amplas áreas de reflorestamento. De acordo com esse autor, nessa mesma ocasião foi verificado que as plantas apresentavam sintomas de quedas de folhas e redução do crescimento provocado pela presença dos insetos, sintomas esses similares aos danos causados por esse mesmo inseto em lavouras de café.

A presença de grandes populações de cigarras vem sendo associada a fatores provocantes de injúria e morte de árvores de paricá. O acompanhamento da demografia desse inseto é uma prática corriqueira nos reflorestamentos localizados nas proximidades do Município de Dom Eliseu, PA.

As populações de insetos em plantações podem ser mapeadas através do uso de procedimentos de interpolação, que permitem gerar superfícies contínuas através de unidades amostrais pontuais. Alguns procedimentos se baseiam em relações matemáticas simples, como o método de interpolação do inverso do quadrado da distância, em que os valores da infestação em pontos não amostrados são calculados a partir de uma relação entre as distâncias e os valores de pontos vizinhos amostrados. Procedimentos mais aplicados levam em consideração, além da distância entre os pontos não-amostrados e os pontos amostrados, o estabelecimento de um modelo para a dependência espacial para a variável em estudo. Tais procedimentos são conhecidos como geoestatísticos, entre os quais está incluída a krigagem ordinária (ISAAKS e SRIVASTAVA, 1989).

A escolha de um método de intepolação ou de um modelo de dependência espacial pode ser feita através da validação cruzada (ISAAKS e SRIVASTAVA, 1989), erro médio e raiz quadrada do erro médio (MURPHY e STERNITZKE, 1979). Para realizar a validação cruzada, o valor observado em cada ponto amostrado é temporariamente removido e é calculada uma estimativa para o ponto amostrado, usando-se os pontos amostrados adjacentes através de um método de interpolação. Ao final, para cada ponto amostrado têm-se o valor observado e uma estimativa. A validação cruzada se baseia na regressão dos valores observados versus os estimados. Quando as estimativas dos parâmetros $\beta_{1}$ e $\beta_{0}$ dessas regressões são, respectivamente, próximas de 1 e 0 , a relação entre os valores preditos e observados é alta, e conclui-se que o método de interpolação ou o modelo de dependência espacial é adequado (ISAAKS e SRIVASTAVA, 1989).

O desvio, entre o valor observado e o estimado, é definido como o erro ou resíduo de estimação. A partir desses resíduos são calculados a média dos erros e a raiz quadrada do erro médio. O sinal do erro médio sugere a tendência dos resultados em superestimar (quando positivo) ou subestimar (quando negativo) o valor da variável em estudo. No entanto, o valor ideal da raiz quadrada do erro médio é igual a 1. Quanto maior o valor da raiz quadrada do erro médio, mais intensas as derivas entre os valores estimados e seus valores verdadeiros (MURPHY e STERNITZKE, 1979).

Alguns autores já vêm aplicando geoestatística para estudar a distribuição espacial de insetos em cultivos comerciais. Gilbert et al. (2001) analisaram a presença do inseto Dendroctonus micans em plantações de abeto (árvore da família das Pináceas), através de procedimentos geoestatísticos. Neste trabalho, os autores não tiveram sucesso na detectação de dependência espacial nos dados de suas amostras. Isaaks e Srivastava (1989) sugeriram que, mesmo que os dados amostrais não demonstrem um modelo de continuidade espacial claramente definido, não se justifica o uso de modelos de funções aleatórias não correlacionadas espacialmente. Esses autores ressaltaram que, geralmente no tocante aos dados relativos ao ambiente, é possível modelar a continuidade espacial. Contudo, é possível que essa continuidade não esteja evidente nas amostras disponíveis, devido ao seu número insuficiente, aos erros de amostragem ou à excessiva presença de dados discrepantes.

Os resultados obtidos por Gilbert et al. (2003) corroboram essa hipótese, pois diferentemente do reportado por Gilbert et al. (2001), foi constatada alta relação espacial da distribuição espacial da mesma praga. No entanto, Gilbert et al. (2003) levaram em consideração, na modelagem da continuidade espacial, fatores ambientais relacionados à presença do inseto.

Baseado nas observações e implicações mencionadas anteriormente, este trabalho teve como objetivo geral avaliar o uso de diferentes métodos de 
interpolação do número de insetos, com a finalidade de analisar a precisão dos procedimentos atuais de mapeamento das populações de cigarras em plantios de paricá.

\section{METODOLOGIA}

\subsection{Localização da área de estudo}

A área de estudo encontra-se em um reflorestamento de paricá localizado no Município de Dom Eliseu, PA, com coordenadas UTM (210.000, 9.551.500), zona 23 M. Na época da coleta de dados, a propriedade em questão apresentava plantios de paricá com diferentes idades e distribuídos em talhões de tamanhos variáveis, com divisão não uniforme devido às propriedades da geografia local (Figura 1).

\subsection{Base de dados, softwares e definição das unidades amostrais}

Os arquivos de mapeamento da empresa proprietária da área de estudo foram utilizados para se criar uma base de dados no formato geodatabase do programa ArcGIS. A base de dados foi composta de elementos lineares e poligonais, os quais definiram, respectivamente, as estradas e os talhões dentro do reflorestamento.

Os plantios usados neste trabalho tinham paricá com idades de 5 e 6 anos. A amostragem seguiu os padrões adotados pelas empresas locais, com uma unidade amostral a cada 30 ha. A distância entre as unidades amostrais mais próximas foi em torno de $400 \mathrm{~m}$. Nessa área foram utilizadas 31 unidades amostrais, sendo cada unidade amostral constituída por uma única árvore (Figura 1).

As unidades amostrais foram selecionadas aleatoriamente com o uso do software ArcGIS. Posteriormente, as unidades amostrais foram localizadas na área em estudo, com GPS de navegação, utilizandose as suas coordenadas.

\subsection{Contagem e registro do número de cigarras}

Em cada unidade amostral foi registrado o número de cigarras por árvore. Para isso, foi delimitada uma área comum ao redor da árvore amostrada, dentro da qual foram feitas uma limpeza e a posterior contagem do número de cigarras. Essa contagem visava quantificar o número de insetos que estavam se alimentando do sistema radicular em cada unidade amostral. A área comum abrangeu um círculo de $1,5 \mathrm{~m}$ de raio ao redor da planta amostrada, em que uma camada superficial de mais ou menos $5 \mathrm{~cm}$ de solo foi removida para descobrir os orifícios onde estavam localizadas as ninfas.

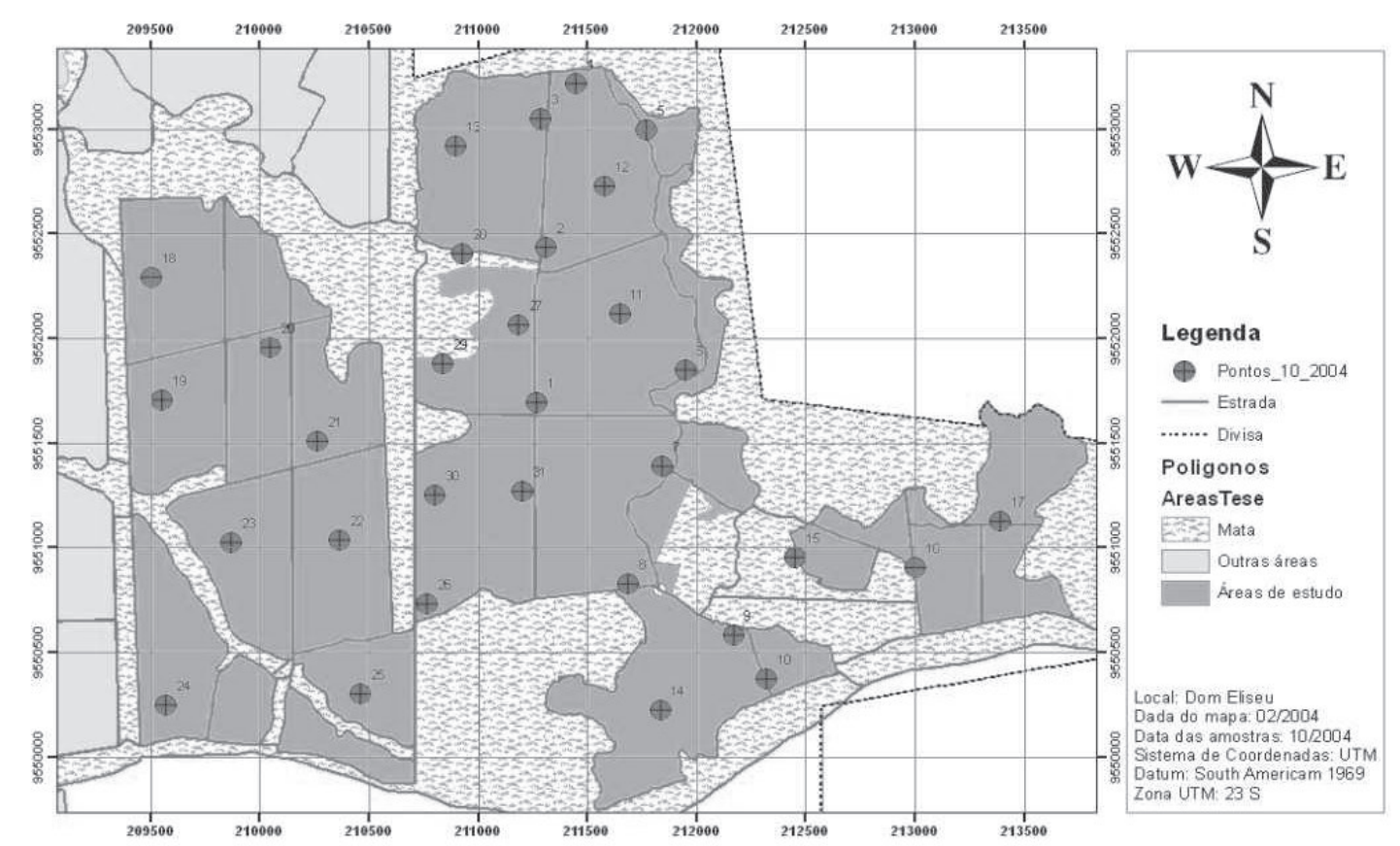

Figura 1 - Fazenda Chapadão de Dom Eliseu, PA, mostrando as 31 unidades amostrais. Figure 1 - Chapadão de Dom Eliseu Farm, showing the 31 sampling units. 


\subsection{Geração dos mapas de dispersão de cigarras}

Através da extensão Geostatistical Analyst do software ArcGIS, foram gerados os mapas do número de cigarras/árvore, através dos métodos de interpolação do inverso do quadrado da distância e da krigagem ordinária.

Durante a utilização do interpolador do inverso do quadrado da distância, foi necessário estabelecer os valores do raio de busca e da quantidade de pontos amostrados vizinhos que seriam usados na interpolação. O raio de busca determina a distância de influência de um ponto amostrado sobre a estimativa de pontos não amostrados. O número máximo define um limite superior de vizinhos, para os casos em que, dentro do raio de busca, existam muitos pontos amostrados passíveis de serem usados na estimativa do valor de um ponto não amostrado. Já o número mínimo define um limite inferior de pontos amostrados vizinhos, necessários para estimar o valor de um ponto não amostrado. Caso não exista um número mínimo de vizinhos dentro do raio de busca, o algoritmo procura vizinhos fora do raio de busca. Neste trabalho, o raio de busca e os números máximo e mínimo de pontos vizinhos foram definidos como $2.000 \mathrm{~m}$ e $16 \mathrm{e} 3$ pontos vizinhos, respectivamente, seguindo-se os valores sugeridos pelos trabalhos anteriormente efetuados pela empresa fornecedora dos dados.

Para a aplicação do método da krigagem ordinária, inicialmente foi selecionado o modelo de semivariograma (circular, esférico, exponencial e gaussiano), que apresentou os melhores resultados na validação cruzada. Posteriormente, o melhor modelo foi utilizado para gerar os mapas do número de cigarras por unidade amostral. Nessa interpolação, não houve necessidade de definição do raio de busca para pontos vizinhos, pois o raio de busca é automaticamente definido pelo alcance do modelo de semivariograma ajustado aos dados. Os valores máximos e mínimos de vizinhos foram definidos da mesma forma que para o método do inverso do quadrado da distância.

\subsection{Avaliação dos métodos de interpolação e das estratégias de amostragem}

Os resultados da validação cruzada também foram utilizados para comparar os métodos de interpolação inversos do quadrado da distância e krigagem. Foi obtido para cada ponto amostrado um par de valores constituídos pelo valor observado e o estimado e os respectivos resíduos calculados pela diferença entre o valor observado e o respectivo valor estimado. Um dos resultados da validação cruzada utilizados foi o ajuste do modelo de regressão linear de primeiro grau dos valores observados em função dos valores estimados. Quanto mais próximas de 1 e 0 forem as estimativas obtidas para $\beta_{1}$ e $\beta_{0}$, respectivamente, melhor será o método de interpolação ou modelo de dependência espacial (ISAAKS e SRIVASTAVA, 1989). Os valores obtidos, com a validação cruzada, para o erro médio e a raiz quadrada do erro médio também foram utilizados. Para esses dois parâmetros, os resultados desejados foram: erro médio próximo de zero e raiz quadrada do erro médio com valor próximo de 1 (MURPHY e STERNITZKE, 1979).

Os parâmetros acima descritos foram usados para se decidir a respeito da qualidade dos métodos de interpolação e, mesmo, entre os modelos de semivariograma ajustados às quantidade de cigarras/ árvores amostradas neste estudo.

\section{RESULTADOS E DISCUSSÃO}

Nos 31 pontos amostrados (Figura 1), o número de cigarras/árvore variou de 0 a 67, indicando elevada amplitude dos valores observados no número de cigarras/ árvore na área estudada, como pode ser observado no Quadro 1.

Os ajustes dos modelos de semivariograma exponencial, esférico, circular e gaussiano indicaram existir fraca dependência espacial para o número de cigarras/árvore (Figura 2). Todos os modelos apresentaram valores muito reduzidos para o alcance, se comparados com a distância mínima entre pontos (em torno de $400 \mathrm{~m}$ ), havendo poucos pares de pontos na parte curva do semivariograma, ou seja, antes de atingir o alcance. Exceto para o modelo exponencial, o valor do efeito-pepita tendeu sempre a apresentar valor elevado. De acordo com Isaaks e Srivastava (1989), à medida que o valor do efeito-pepita aumenta e, conseqüentemente, se aproxima do valor do patamar, a dependência espacial enfraquece, pois os pesos dos diferentes pontos amostrados, usados na estimativa de dado ponto não amostrado, tornam-se semelhantes. Portanto, os resultados encontrados neste trabalho indicam fraca dependência espacial para o número de cigarras/árvore. 
Quadro 1 - Número de cigarras/árvore observado em cada unidade da amostragem realizada em outubro de 2004 Table 1 - Number of buzzers/tree observed in each sampling unit on October 2004

\begin{tabular}{|c|c|c|c|c|c|c|c|}
\hline \multirow{2}{*}{$\begin{array}{l}\text { unidades } \\
\text { amostrais }\end{array}$} & \multicolumn{2}{|c|}{ Coordenadas } & \multirow{2}{*}{$\begin{array}{c}\text { NÚMERO de } \\
\text { cigarras / árvore }\end{array}$} & \multirow{2}{*}{$\begin{array}{c}\text { unidades } \\
\text { amostrais }\end{array}$} & \multicolumn{2}{|c|}{ Coordenadas } & \multirow{2}{*}{$\begin{array}{c}\text { NÚMERO de } \\
\text { cigarras / árvore }\end{array}$} \\
\hline & $\mathrm{X}$ & $\mathrm{Y}$ & & & $\mathrm{X}$ & $\mathrm{Y}$ & \\
\hline 1 & 210741 & 9551823 & 67 & 17 & 211453 & 9553219 & 6 \\
\hline 2 & 210942 & 9552408 & 58 & 18 & 211775 & 9552994 & 0 \\
\hline 3 & 209509 & 9552292 & 1 & 19 & 211952 & 9551848 & 15 \\
\hline 4 & 209556 & 9551703 & 0 & 20 & 211849 & 9551391 & 35 \\
\hline 5 & 210052 & 9551957 & 5 & 21 & 211690 & 9550828 & 6 \\
\hline 6 & 210271 & 9551509 & 0 & 22 & 212172 & 9550581 & 0 \\
\hline 7 & 210373 & 9551038 & 1 & 23 & 212322 & 9550374 & 0 \\
\hline 8 & 209872 & 9551026 & 1 & 24 & 211655 & 9552119 & 4 \\
\hline 9 & 209578 & 9550250 & 0 & 25 & 211586 & 9552725 & 0 \\
\hline 10 & 210467 & 9550299 & 0 & 26 & 210900 & 9552919 & 4 \\
\hline 11 & 210773 & 9550732 & 15 & 27 & 210809 & 9551251 & 53 \\
\hline 12 & 211208 & 9551270 & 3 & 28 & 211838 & 9550222 & 5 \\
\hline 13 & 211274 & 9551696 & 42 & 29 & 212455 & 9550953 & 2 \\
\hline 14 & 211191 & 9552066 & 18 & 30 & 213004 & 9550908 & 3 \\
\hline 15 & 211314 & 9552433 & 11 & 31 & 213392 & 9551125 & 0 \\
\hline 16 & 211292 & 9553049 & 26 & & & & \\
\hline
\end{tabular}
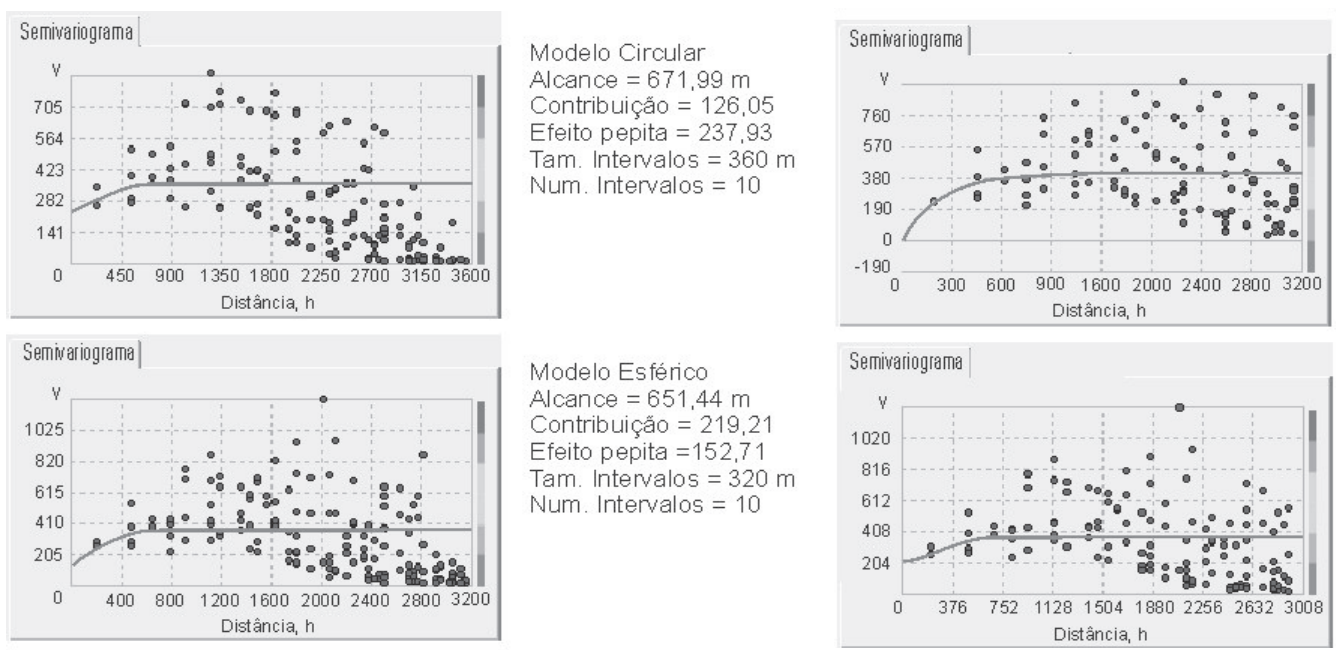

Modelo Exponencial Alcance $=677.51 \mathrm{~m}$ Contribuição $=413,97$ Efeito pepita $=0$ Tam. Intervalos $=300 \mathrm{~m}$ Num. Intervalos = 8

Figura 2 - Ajustes dos modelos de semivariogramas usados para gerar os mapas de krigagem.

Figure 2 -Fitting of the semi-variogram models used to generate the kriging maps.

Como observado por Gilbert et al. (2001), para melhorar a dependência espacial pode ser necessário efetuar uma análise espacial mais ampla, envolvendo mais variáveis relacionadas à presença do inseto na área. Além disso, a quantidade de pontos provavelmente esteja sendo insuficiente para ajustar bem os modelos de semivariograma, como mencionado por Isaaks e Srivastava (1989), e talvez seja necessário pensar em métodos alternativos que permitam maior intensificação da amostragem, resultando em mais pontos usados no ajuste dos semivariogramas.

No estudo da validação cruzada dos modelos de semivariograma ajustados, observou-se que, para todos os modelos, a relação entre os valores preditos e observados tendeu a ser baixa, pois o coeficiente de regressão $\beta_{1}$ tendeu a apresentar valor muito baixo, em torno de 0,33 , e o $\beta_{0}$ bastante alto, em torno de 7 (Figura 3), ao contrário do que seria desejável, $\operatorname{com} \beta_{1}$ próximo de 1 e $\beta_{0}$ próximo de zero, como sugerido por Isaaks e Srivastava (1989).

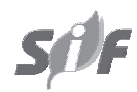

R. Árvore, Viçosa-MG, v.32, n.2, p.251-258, 2008 


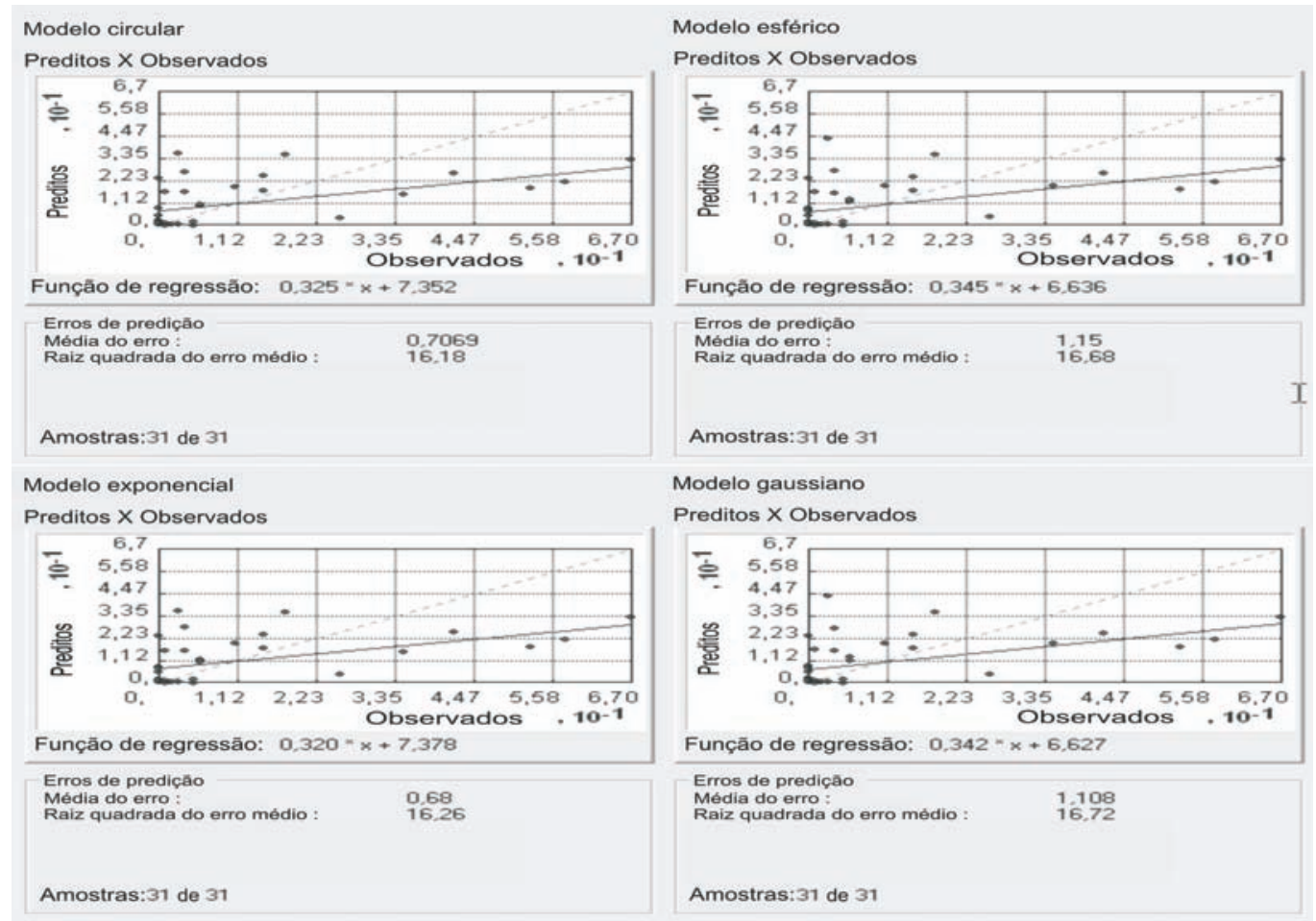

Figura 3 - Validação cruzada dos mapas de predição do número de cigarras/árvore obtidos ao ajustar quatro modelos de semivariograma. Figure 3 -Crossed validation for the prediction maps of number of buzzers /tree obtained from the four semi-variogram models.

A raiz quadrada do erro médio (RQEM) foi bem semelhante para todos os semivariogramas, oscilando em torno do valor 16. Essa similaridade indica que houve pouca diferença entre os modelos de semivariogramas utilizados para representar a dependência espacial do número de cigarras/árvore. O valor 16 para o RQEM, que é relativamente distante do valor ideal igual a 1 , de acordo com Murphy e Sternitzke (1979). Esses resultados reforçam o discutido anteriormente de que o procedimento de amostragem não foi capaz de detectar, de forma precisa, a dependência espacial para o número de cigarras/árvore.

Os mapas gerados usando krigagem ordinária quando os modelos de semivariogramas esférico, exponencial, circular e gaussiano foram utilizados para modelar a dependência espacial do número de cigarras/ árvore - indicam não haver diferenças relevantes entre eles (Figura 4). Em geral, esses mapas revelaram a presença de um foco de cigarras na região central da área de estudo, onde os valores excederam a quantia de 20 insetos por planta. À medida que se distancia desse foco central, houve uma tendência de redução na quantidade de insetos por planta. No entanto, os mapas gerados ao ajustar os modelos de semivariogramas esférico e exponencial apresentaram uma intensidade de praga mais elevada no centro do foco, com um pico mais saliente, se comparado com o mapa gerado ao ajustar o modelo gaussiano (Figura 4).

Apesar de a dependência espacial ter sido fraca, foi escolhido o modelo exponencial para comparar os dois métodos de interpolação, krigagem ordinária e inverso do quadrado da distância quanto à distribuição espacial do número de cigarras/árvore. Tal escolha ocorreu porque o modelo exponencial é indicado para fenômenos de transição espacial rápida, que se julga ser o caso para o número de cigarras/árvore nos reflorestamentos de paricá.

O mapa do número de cigarras/árvore, obtido ao aplicar o método do inverso do quadrado da distância, é apresentado na Figura 5, juntamente com o mapa gerado usando-se a krigagem ordinária a partir do modelo 
exponencial. Visualmente, pode-se constatar que os mapas são muito semelhantes. A semelhança entre os resultados dos dois métodos pode ter sido ocasionada pela fraca dependência espacial detectada na amostragem.
Na ausência de dependência espacial, as vantagens da krigagem sobre o método do IQD são também minimizadas, e os métodos tendem a se equivaler, como sugerido por Isaaks e Srivastava (1989).
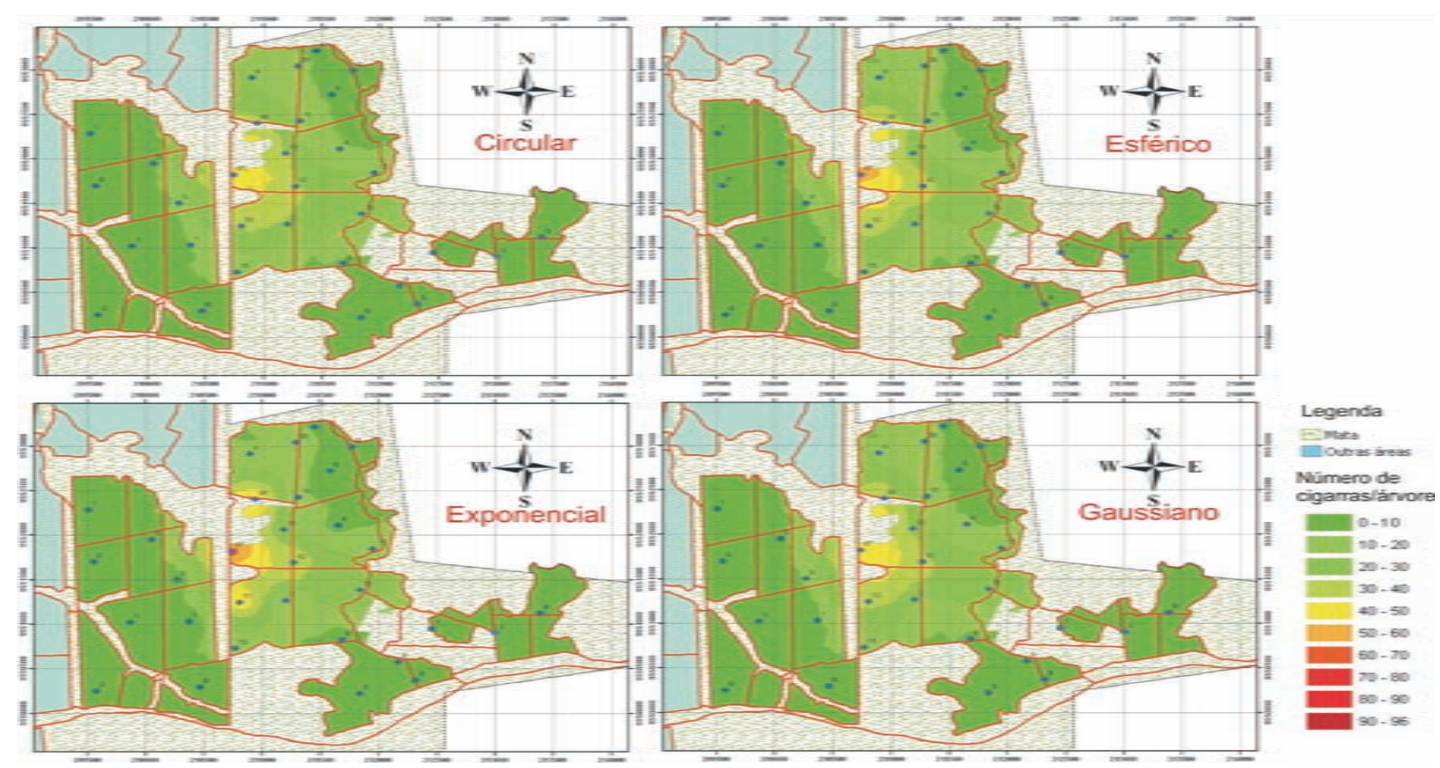

Figura 4 - Mapa de predição por krigagem ordinária do número de cigarras/árvore ao ajustar quatro modelos de semivariograma. Figure 4-Prediction map generated by ordinary kriging showing number of buzzers/tree after adjusting the four semivariogram models.

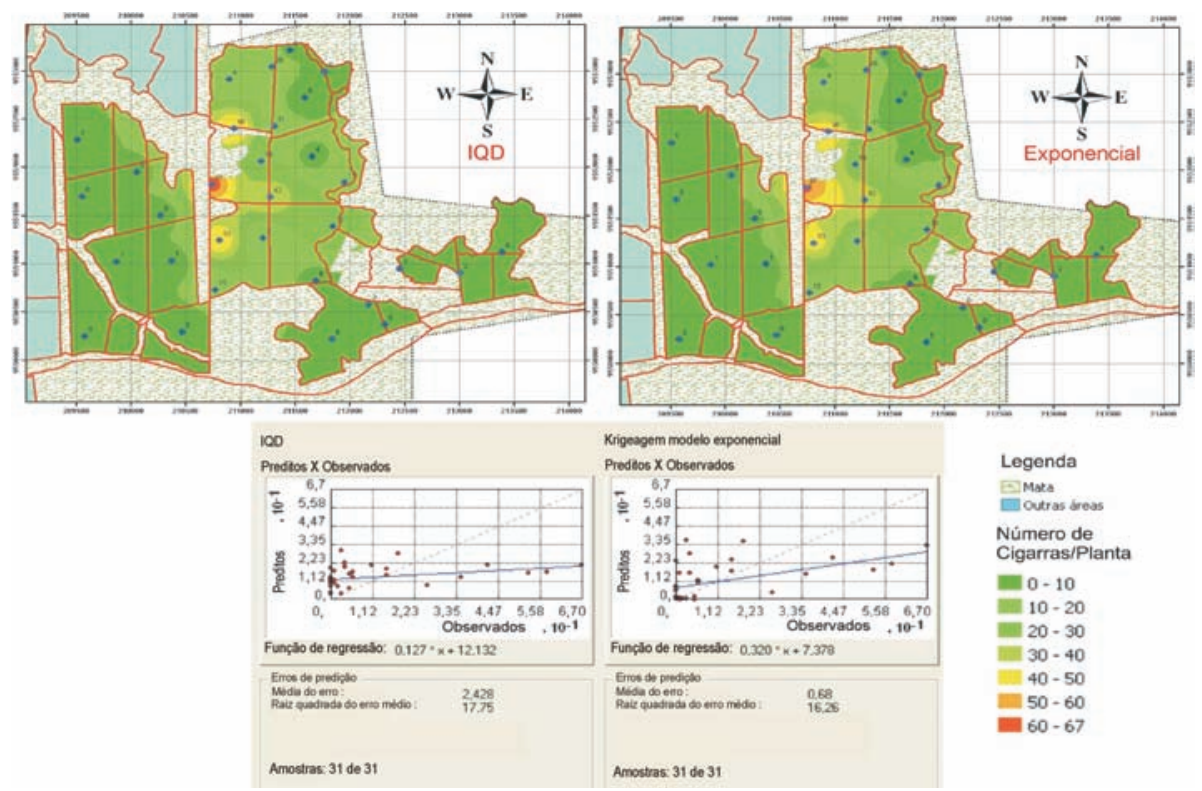

Figura 5 - Mapas de predição do número de cigarras/árvore gerados utilizando o método IQD e da krigagem ordinária (modelo exponencial), com os resultados das respectivas validações cruzadas.

Figure 5 - Prediction maps of the number of buzzers/tree generated by the IQD and ordinary kriging (exponential model) with the results of their crossed validation. 
Apesar da fraca dependência espacial encontrada, os resultados da validação cruzada revelam ligeira superioridade da krigagem sobre o IQD, visto que o $\beta_{1}$ para a krigagem foi de 0,320 contra 0,127 do IQD, sendo superior em mais que o dobro, como apresentado nos respectivos gráficos da validação cruzada apresentados na Figura 5. Observa-se também, nessa figura, que o $\beta_{0}$ e a RQEM da krigagem tenderam a ser menores que os do IQD, bem como a média do erro (ME), que foi de 0,68 no método da krigagem, enquanto no método do IQD ela foi de 2,418 , indicando que o método IQD tendeu a superestimar mais o número de cigarras/árvore que a krigagem ordinária (Figura 5).

\section{CONCLUSÕES}

A intensidade amostral de um ponto a cada 30 ha demonstrou-se insuficiente para detectar dependência espacial do número de cigarras/árvore, o que tende a invalidar procedimentos de amostragem pouco intensos.

O número reduzido de unidades amostrais pode ter influenciado a detecção da dependência espacial, aumentando o poder de influência de observações discrepantes e levando a rápida perda de continuidade do semivariograma.

Dadas as circunstâncias da dependência entre os dados, a krigagem ordinária apresentou mínimas vantagens sobre o método do IQD na geração dos mapas de dispersão de cigarras nos povoamentos de paricá.

Os mapas de dispersão de cigarras em povoamentos de paricá na região de Dom Eliseu, PA, obtidos a partir dos planos de amostragem adotados neste trabalho, estão sendo produzidos com baixa precisão e podem não ser adequados para tomadas de decisões importantes.

\section{AGRADECIMENTOS}

Ao Centro de Pesquisa do Paricá, de Dom Eliseu, Pará, pelo apoio em todas as etapas da condução deste trabalho.

\section{REFERÊNCIAS}

CÂMARA, G.; MONTEIRO, A. M. V.; CARVALHO, S. D. M. S. Análise espacial e geoprocessamento. São José dos Campos, INPE, 2002. 106p.

GILBERT, M.; GRÉGOIRE, J. C. Site condition and predation influence a bark beetle's success: a spatially realistic approach. Agricultural and Forest Entomology, v.5, n.2, p.87, 2003.

GILBERT, M.; VOULAND, G.; GRÉGOIRE, J. C. Past attacks influence host selection by the solitary bark beetle Dendroctonus micans.

Ecological Entomology, v.26, n.2, p.133, 2001.

ISAAKS, E. H.; SRIVASTAVA, R. M. An

introduction to applied geostatistics. New York: Oxford Universit, 1989. 561p.

MURPHY, P. A.; STERNITZKE, H. S. Growth and yield for loblolly pine in West Gulf. U.S. Dep. Agric. For. Serv. Res., Pap. SO154, 8 p. 1979.

ZANUNCIO, J. C. et. al. Occurrence of Quesada gigas (Oliver) on Schizolobium amazonicum (Huber ex Ducke) trees in Maranhão and Pará States, Brazil. Pesquisa Agropecuária Brasileira, v.39, n.9, p.943-945, 2004. 